\title{
UNA VARIANTE PEDAGÓGICA DE LA INVESTIGACIÓN-ACCIÓN EDUCATIVA
}

\author{
Bernardo Restrepo Gómez \\ Coordinador del Consejo Nacional de Acreditación de Colombia
}

\section{INTRODUCCIÓN}

Entre 1998 y 2002 se ha venido realizando en Antioquia, Colombia, con docentes de los niveles de educación preescolar, básica, media y superior, un proyecto de investigación que a finales del año 2000 fue apoyado por COLCIENCI-AS, instituto encargado de promover la política de Ciencia y Tecnología en el país. El proyecto tiene como propósito central probar la viabilidad y la efectividad de la investigación-acción desarrollada por maestros y aplicada particularmente a la transformación de la práctica pedagógica personal de los mismos y proponer un modelo de capacitación de maestros en servicio, basado en la investigación. El proyecto nació en la Escuela de Pedagogía de la Asociación de Colegios Privados de Antioquia que ha servido como animadora de toda la experiencia y poco a poco ha ido configurando una variante especial de la investigación-acción.

Las aplicaciones de la investigación-acción son múltiples, como amplio y variado el espectro investigativo que se encuentra en la literatura relacionada. Es por ello que es benéfico dar una mirada a las aplicaciones particulares que de la misma se han hecho en educación para diferenciarlas de la variante desarrollada en este programa entre 1998 y 2002. Para observadores expertos en investigación-acción educativa (I-AE) debemos decir que la característica sobresaliente de esta variante es la investigación de la práctica pedagógica individual de cada docente.

En este artículo presentaremos primero una breve historia de la $\vdash A$, luego expondremos la variante construida durante cinco años y medio de aplicación de la metodología de $\mathrm{tA}$ a la transformación de la práctica pedagógica de los maestros con sus fases y secuencia, y finalmente comentaremos nuestra posición en favor de la hipótesis del maestro investigador, discutiendo la posibilidad de enseñar e investigar simultáneamente.

\section{BREVE HISTORIA DE LA INVESTIGACIÓN-ACCIÓN Y TIPOS DE APLICACIÓN}

Los antecedentes teóricos de la I-A pueden situarse en el advenimiento del método de investigación-acción propuesto por el sicólogo social Kurt Lewin en la década del 40 (Kemmis y Mctaggart, 1993; Elliot, 1994). Lewin concibió este tipo de investigación como la emprendida por personas, grupos o comunidades que llevan a cabo una actividad colectiva en bien de todos, consistente en una práctica reflexiva social en la que interactúan la teoría y la práctica con miras a establecer cambios apropiados en la situación estudiada y en la que no hay distinción entre lo que se investiga, quien investiga y el proceso de investigación. 
La I-A tuvo desde Lewin varios desarrollos con teorías sociales fundantes diversas y con aplicaciones también diferentes que fluctúan entre la $\mathrm{A} \mathrm{participativa} \mathrm{(I-AP),} \mathrm{la} \mathrm{I-AE,} \mathrm{ligada} \mathrm{a} \mathrm{indagación} \mathrm{y}$ transformación de procesos escolares en general, y la investigación-acción-pedagógica (I-A-Pedagógica), más focalizada en la práctica pedagógica de los docentes. La primera ha sido desarrollada por la sociología comprometida, principalmente desde la década del 60, mientras que la segunda y tercera aparecieron en la década del 50. Nos limitaremos a explicar y ejemplificar aquí solamente las dos últimas aplicaciones, dejando claro desde ahora que este proyecto opta conscientemente por la I-AE de corte pedagógico.

El inicio del primer tipo o primera aplicación a la educación puede situarse a finales de la década del 40 cuando Stephen Corey y otros lanzaron en la Universidad de Columbia -donde Kurt Lewin había abogado por la I-A- el movimiento por un maestro investigador. En 1953 Corey, profesor del Teachers'College of Columbia University, publicó, junto con otros profesores de esta universidad, una obra sobre I-A como método para mejorar las prácticas escolares. Corey concibió este método como aquellos procesos investigativos conducidos por grupos de maestros en su escuela tendientes a comprender su práctica educativa y transformarla. Más concretamente la definió como el «estudio realizado por colegas, en un ambiente escolar, de los resultados de actividades para mejorar la instrucción». Este profesor de la Universidad de Columbia, influenciado por el pensamiento de su colega Kurt Lewin, hizo hincapié en as conexiones existentes entre la investigación social y los movimientos sociales de la época. Aunque fue la primera aplicación de la tA a la educación e incluyó la investigación sobre el desarrollo del currículo, todavía no puede hablarse en sí de la aplicación a la transformación de la práctica pedagógica del maestro.

En Inglaterra, antes de Stenhouse, se tuvo esta misma concepción de la rAE. Las primeras alusiones a este tipo de investigación demandaban la participación de todos los miembros de una organización en el desarrollo de la investigación y una cooperación de actores de la realidad, los maestros, y de investigadores de carrera. Esta es la visión planteada por Rapaport en 1970, citado por Parra (1995).

En el mismo sentido Stephen Kemmis, de la Universidad de Deakin, Australia, ha pensado la I-A como actividad colectiva que propende la transformación de procesos educativos asociados a procesos sociales y en definitiva por el mejoramiento social (Kemmis and Mctaggart, 1988).

El segundo tipo o segunda aplicación, puede remontarse, en forma más contundente, a la década del 70 cuando Stenhouse, reformador del currículo de las humanidades en Inglaterra, clamó por una investigación educativa naturalística, no positivista, centrada en el interior de la escuela y de los procesos educativos y realizada por los practicantes de la educación, los maestros (Stenhouse, 1993). Su alumno y colaborador, John Elliot, ha continuado esta línea y ha publicado una obra sobre La Investigación-Acción en Educación (1994) en la que fundamenta esta propuesta. Elliot subraya que la łA aplicada a la educación tiene que ver con los problemas prácticos cotidianos experimentados por los docentes, más que con problemas teóricos definidos por investigadores dentro de un área del conocimiento.

Según Stenhouse y Elliot (1993; 1994), la docencia no es una actividad realizada por los maestros, y la investigación sobre la enseñanza otra actividad llevada a cabo por investigadores externos y de otras 
disciplinas. Esta separación entre investigadores y maestros ha sido la situación predominante en el pasado. A este respecto Stenhouse, analizando en su obra Investigación y desarrollo del currículo (1981) enfoques de la investigación en el aula, afirma:

La mayor parte del trabajo realizado en esta área (investigación sobre la enseñanza) se ha basado en observadores que eran más investigadores que maestros. $Y$ en general dichos investigadores se han interesado más por construir una teoría sobe la enseñanza y comunicar observaciones, en una forma dirigida sobre todo a la comunidad de investigadores, que en mejorar las aulas que han estudiado. No puede afirmarse esto de toda la obra que se ha publicado, pero siempre existen, al menos huellas de la separación entre investigadores y profesores.

Frente a esta situación Stenhouse (ibidem, p. 210) propone integrar en el docente los tres roles de investigador, observador y maestro. Al respecto afirma:

En mi concepto esto es perfectamente posible, siempre y cuando el profesor ponga en claro que la razón por la que está desempeñando el papel de investigador es la de desarrollar positivamente su enseñanza y hacer mejor las cosas.

En la misma obra Stenhouse, al considerar el concepto de «profesionalidad amplia» del docente, esencial para una investigación y un desarrollo bien fundamentado del currículo, plantea que las características de tal profesionalidad son:

El compromiso de poner sistemáticamente en cuestión la enseñanza impartida por uno mismo como base de desarrollo.

El compromiso y la destreza para estudiar el propio modo de enseñar.

El interés por cuestionar y comprobar la teoría en la práctica mediante el uso de dichas capacidades (Ibid., p. 197).

Al considerar la práctica de la enseñanza como una actividad reflexiva que se orienta a hacer mejor las cosas, la división del trabajo entre practicantes e investigadores se desvanece y se prepara a los practicantes para asumir ambas tareas como una integración, aunque Stenhouse valora el acompañamiento de investigadores externos a esta labor de los practicantes de la educación. La investigación educativa en las aulas aparece, entonces, como alternativa a la investigación sobre educación y como una característica de una profesionalidad ampliada del docente frente a la profesionalidad restringida que generalmente han ejercido los maestros, esto es, una profesionalidad limitada a poner en práctica teorías e investigaciones hechas por otros.

A esta posición de Stenhouse hacemos una glosa fundamentada en observaciones que provienen de los proyectos de las dos primeras cohortes de maestros investigadores de ADECOPRI-A: si bien es cierto que los maestros activos pueden enfrentar con ventajas la investigación de la práctica docente, en tanto pedagogía, también lo es que no es posible enderezar las deficiencias de la práctica de enseñanza sin un dominio adecuado del saber que se enseña y sin un diálogo entre el método en sí de dicho saber y el método didáctico a través del cual aquel se enseña. Es necesario superar tanto el pedagogismo como el 
cientifismo. Esto implica atender en los proyectos de I-AE la lectura y la formación en el saber cuya enseñanza se intenta transformar.

En la posición de Stenhouse y Elliot aparece una meta diferente: la enseñanza, la práctica educativa o, si se quiere, la $\mathrm{HAE}$, que toma distancia de la I-A sobre educación, más centrada en las relaciones de la educación con los procesos sociales paralelos a la misma. La I-A-pedagógica, pensamos, se centra en los microprocesos de clase, en el desarrollo del currículo como objeto primordial. Tal el énfasis que Stenhouse pone en su obra principal Investigación y desarrollo del currículo (1981).

En el proyecto que se ha desarrollado en ADECOPRI-A desde 1998, y de manera más investigativa con la cofinanciación de COLCIENCI-AS entre 2000 y 2002, se defiende la tesis de la investigación sobre problemas individuales, sobre la práctica pedagógica personal del maestro, aunque el primer ciclo se lleva a cabo en forma participativa. Si cada docente identifica su problema y trabaja todo el proyecto alrededor del mismo, la discusión y crítica de la fase de deconstrucción o reflexión sobre la práctica pedagógica anterior y actual se hace en el seno de un grupo de colegas que a su vez trabajan sus propios proyectos y que se ayudan. Hay, entonces, un proceso de crítica social al develar los problemas de la práctica particular, pero ciertamente no se atacan problemas colectivos ni se intentan transformaciones de índole social. Si bien la transformación no es colectiva, de grupo, es investigación porque sigue los parámetros de búsqueda sistemática de conocimiento y es investigación-acción porque se pretende una transformación de la práctica pedagógica durante el desarrollo mismo del proyecto.

Donald Schon, orientado en la misma dirección de la investigación-accion pedagógica, expone en 1983 su teoría de la «práctica reflexiva» o «enseñanza reflexiva», en la cual aboga por un maestro que reflexione permanentemente sobre su práctica de enseñanza con el fin de transformarla. Más tarde, en una presentación en la Convención de la Asociación Americana de Investigación en Educación, reafirma esta posición y defiende la opción de que el maestro construya saber pedagógico a través de la "reflexión en la acción» y se separe del discurso pedagógico oficial aprendido en las Facultades de Educación (1987). Su objetivo está muy cerca del de la I-AE-pedagógica, sólo que ésta tiene un propósito más investigativo, más sistemático, con identificación de un problema de la práctica, que se somete a la lógica abierta de la investigación científica.

Por su objeto, entonces, la $\mathrm{HAE}$ puede enfocarse a transformar instituciones escolares totales o unidades o prácticas sociales de la misma, o puede circunscribirse a la práctica pedagógica de un docente particular.

Por sus actores, los proyectos investigativos pueden ser colaborativos, colectivos, o individuales. 


\section{VARIANTE PARA LA INVESTIGACIÓN DE LA PRÁCTICA PEDAGÓGICA. AFINIDAD Y DIFERENCIA CON OTRAS VARIANTES DE I-A}

\subsection{Particularidades de la I-A-pedagógica}

El modelo orientador de este trabajo es el modelo básico de la investigación-acción que incluye en todos los prototipos de ésta tres fases que se repiten una y otra vez, siempre con el fin de transformar la práctica y buscar mejorarla permanentemente. Estas fases son: la reflexión sobre un área problemática, la planeación y la ejecución de acciones alternativas para mejorar la situación problemática, y la evaluación de resultados con miras a emprender un segundo ciclo o bucle de las tres fases. La reflexión, en verdad, se encuentra al comienzo del ciclo, en la planeación y en la evaluación o seguimiento de la acción instaurada para transformar la práctica.

Frente a la participación de investigadores externos en los proyectos de investigación, el modelo seguido considera a los maestros como investigadores que participan en todo el proyecto. Existen uno o varios investigadores acompañantes o animadores del proceso, pero no como actores externos que se basan en el trabajo de los «practicantes» de la educación, los maestros, para daborar análisis y hacer interpretaciones sobre los datos que presentan los practicantes, como suele acontecer en algunos prototipos de la investigación acción. Aquí el maestro investigador es protagonista de primer orden en la formulación, desarrollo y evaluación de su proyecto.

El énfasis de este prototipo de I-A pedagógica está puesto sobre la práctica pedagógica del maestro. No se tienen pretensiones de incidir en el cambio social del contexto inmediato y mucho menos en la transformación radical de las estructuras políticas y sociales del contorno. Se defiende, también, la posibilidad de hacer investigación individual, sin la participación de todo el grupo escolar al que pertenece el maestro, aunque en el primer ciclo de investigación ésta se emprende con un grupo de maestros investigadores, pero sobre proyectos diferentes adelantados por cada investigador participante. El papel de los colegas es ser validadores del trabajo de cada docente investigador, acompañándole con sus comentarios, preguntas, críticas, sugerencias y otros aportes.

El trabajo continuo con varios grupos o cohortes de maestros investigadores ha permitido construir un prototipo de I-A-E particular en el cual la primera fase se ha constituido como una deconstrucción de la práctica pedagógica del maestro, la segunda como una reconstrucción o planteamiento de alternativas y la tercera como evaluación de la efectividad de la práctica reconstruida. Veamos la naturaleza de estas fases en el modelo.

\subsubsection{Deconstrucción}

En el modelo de investigación-acción-educativa seguido por el proyecto de Antioquia se incluye, como se dijo arriba, un proceso de deconstrucción de la práctica, no sólo de reflexión sobre ella. Para llevar 
a cabo este primer paso metodológico, deconstrucción a partir de los datos del diario de campo, con miras a delinear la estructura de la práctica, sus vacíos y elementos de inefectividad, así como las teorías implícitas que la informan, se acudió a los aportes de Stenhouse sobre el enfoque alternativo más atrayente para quienes realizan investigación de aula, el denominado «método social antropológico» (Stenhouse, 1981), y a los del filósofo francés Jacques Derrida sobre deconstrucción de textos como método de indagación analítica (1985).

Stenhouse, al discutir métodos para registrar y analizar los acontecimientos del aula prefiere el método social antropológico sugerido por Walker (1971) consistente en utilizar la observación directa de acontecimientos en el aula, recurriendo a detallados apuntes de campo como medio de registro. La teoría se va construyendo gradualmente a partir del examen de observaciones acumuladas, caracterizando la cualidad de las situaciones particulares.

En cuanto al aporte de Derridá, la intención es utilizar el término «deconstrucción», acuñado por este filósofo francés, para analizar la práctica pasada y presente desde la retrospección, los textos del diario de campo, las observaciones del docente y las entrevistas focales con los alumnos, teniendo en cuenta que unos y otras están mediados por múltiples factores como la cultura, las ideologías, los símbolos, las convenciones, los géneros, la comunicación, que no dejan traslucir directa y transparentemente las ideas de sus autores. Según Mary Klages (1997), Derrida considera la deconstrucción como la puesta en juego de los elementos de la estructura del texto para sacudirla, hallar sus opuestos, atacar el centro que la sostiene y le da consistencia para hallarle las inconsistencias, volverla inestable y encontrarle un nuevo centro que no será estable indefinidamente, pues el nuevo sistema puede contener inconsistencias que habrá que seguir buscando.

El sentido de la investigación-acción educativa, como la practicamos en este proyecto, en efecto, es la búsqueda continua de la estructura de la práctica y sus raíces teóricas para identificarla y someterla a crítica y mejoramiento continuo. Al hablar de la estructura de la práctica nos referimos a que ésta consta de ideas (teoría), herramientas (métodos y técnicas), y ritos (costumbres, rutinas, exigencias, hábitos), susceptibles todos de deconstrucción. El concepto de «deconstrucción» de Derrida, pensado por éste como aplicación al texto escrito, y adaptado aquí a la práctica social y pedagógica del maestro, es de gran utilidad para diagnosticar y criticar la práctica anterior y corriente, utilizando para ello, entre otras técnicas, un diario de campo detallado que privilegia la escritura sobre el discurso oral (Graves, 1998) y que se somete a riguroso examen e interpretación hermenéutica para hallar las bases íntimas de la práctica antes de ensayar alternativas de acción. De especial consideración en los fundamentos teóricos del prototipo de tAE que hemos ido construyendo es el hecho de que la transformación de la propia práctica pedagógica pasa por una pedagogía emancipatoria en el sentido de que el maestro penetra su propia práctica cotidiana, a veces fosilizada, la desentraña, la critica y, al hacer esto, se libera de la tiranía de la repetición inconsciente, pasando a construir alternativas que investiga y somete a prueba sistemática. Se aplica aquí el planteamiento de Edgar Morin (1999) según el cual no solo poseemos ideas, sino que existen ideas posesoras, es decir, ideas que nos poseen y enajenan dirigiendo nuestro pensamiento y acción. De este tenor son las teorías implícitas u operativas que fosilizan nuestra práctica. La crítica y la autocrítica, propias de la $\vdash$, develan estas ideas posesoras y nos permiten desarmarlas. La introspección, el autoexamen 
crítico, nos permite, además, descubrir nuestras debilidades pedagógicas y dejar de asumir siempre la posición de juez en todas las cosas (Morin, 1999, 24). En estos procesos de deconstrucción y reconstrucción, la relación ética educador-educando se revisa y se erige como la relación más destacada de la práctica pedagógica. El reconocimiento de las propias limitaciones, la autocrítica y catarsis de éstas derivada, la comprensión más profunda del proceso pedagógico y sus aristas, la identificación de fuerzas conflictivas que subyacen en la práctica, llevan al docente de la inseguridad y la confusión profesional a la serenidad frente al proceso pedagógico y le permiten dudar sin pánico de los esquemas organizativos de la clase y de los métodos preferidos o simplemente utilizados...

\subsubsection{Reconstrucción}

Con respecto a la reconstrucción, por otro lado, ésta sólo es posible con una alta probabilidad de éxito si previamente se da una deconstrucción detallada y crítica de la práctica. No se trata, tampoco, de apelar a innovación total de la práctica desconociendo el pasado exitoso. Es una reafirmación de lo bueno de la práctica anterior complementada con esfuerzos nuevos y propuestas de transformación de aquellos componentes débiles, inefectivos, ineficientes. Toda investigación tiene como meta la búsqueda y creación de conocimiento. La I-AE lo hace en dos momentos: al deconstruir la práctica o reflexionar sobre la misma críticamente descubre su estructura y los amarres teóricos u operativos de la misma, lo que ya es un conocimiento sistemático; y al reconstruir la práctica se produce saber pedagógico nuevo para el docente y se le objetiva y sustenta por escrito. Todo este proceso consiste en pasar de un conocimiento práctico más bien inconsciente, conocimiento práctico que, como dice Schon, es un proceso de reflexión en la acción o conversación reflexiva con la situación problemática (Schon, 1983), a un conocimiento crítico y teórico. Hay que resaltar aquí, como se hace en otros apartes de este informe, que el objetivo de la FA-E es la transformación de la práctica a través de la construcción de saber pedagógico individual. No se trata de construir teoría general, como bien lo dice Stenhouse $(1981,211)$ :

En consecuencia, el profesor no se enfrenta con los problemas a que da lugar generalizar más allá de la propia experiencia. Dentro de este contexto, la teoría es simplemente una estructura sistemática de la comprensión de su propia labor.

En suma, la IA-E es un instrumento que permite al maestro comportarse como aprendiz de largo alcance, como aprendiz de por vida, ya que le enseña cómo aprender a aprender, cómo comprender la estructura de su propia práctica y cómo transformar permanente y sistemáticamente su práctica pedagógica.

\subsubsection{Evaluación de la práctica reconstruida}

La última fase es la evaluación de la nueva práctica. Para ello se monta ésta y se deja actuar por cierto tiempo, acompañando su accionar con notas sobre indicadores de efectividad. Después de observar sus resultados se analizan las notas del diario de campo y se juzga el éxito de la transformación 


\section{POSICIONES FRENTE A LA POSIBILIDAD DE FORMAR UN MAESTRO QUE INVESTIGUE A LA VEZ QUE ENSEÑA}

Tres posiciones podemos atisbar en el desarrollo reciente de esta corriente teórica discutida por los formadores de formadores. La primera, en sintonía con Stenhouse, Elliot y Kemmis, es que sí se puede ser investigador a la vez que se enseña y se forma, ejerciendo ambas actividades de tiempo completo. Este tipo de investigación se realiza sobre la práctica pedagógica, fundamentalmente.

Una segunda posición, la de quienes consideran la investigación como una práctica rigurosa, sin niveles de aproximación, es que no es posible ser docente de tiempo completo o preferentemente dedicado a la práctica pedagógica e investigador al mismo tiempo. Entre quienes así piensan está, por ejemplo, Aracelly de Tezzanos, educadora uruguaya ampliamente conocida en nuestro medio. La actividad investigativa implica tal responsabilidad y seguimiento de procesos tan complejos que no es dable combinar las responsabilidades de la docencia y de la investigación.

La tercera posición, la nuestra en verdad, es en cierto modo intermedia, conciliadora. Sí y no. No es dable investigar sobre los objetos de los saberes específicos que se enseñan, al menos hacer investigación útil que aporte resultados significativos al cuerpo de conocimientos existente, porque no se cuenta con el equipo requerido ni con el tiempo indispensable para ello y quizás tampoco con la preparación especializada. El docente puede estudiar y debe estudiar e saber que enseña y leer investigación al respecto para incorporarla a su enseñanza, pero ello no constituye investigación de tal saber. Sí es dable investigar sobre la propia práctica pedagógica, bien sea sobre la enseñanza o bien sobre la formación, porque sobre estos menesteres el practicante de la educación tiene a la mano los datos, tiene la vivencia, puede utilizar la retrospección, la introspección y la observación de participante para elaborar relaciones, especificarlas, clarificarlas, comparar teorías guías e intervenciones pedagógicas que permitan resignificar y transformar prácticas no exitosas. Los físicos observan el universo. Galileo construyó un telescopio y descubrió las manchas solares. El maestro observa el universo de su práctica pedagógica y descubre las manchas que le impiden ser más efectivo en su enseñanza, consigna por escrito tales observaciones y críticas, ensaya y valida sistemáticamente sus propuestas de transformación y genera saber pedagógico.

\section{REFERENCIAS}

COREY, Stephen (1953): Action Research to improve school practices. Nueva York, Teachers' College Press.

DERRIDA, Jack (1985): «Letter to a Japanese Friend», en Wood y Bernasconi (eds.): Derrida and Differance. Warvich, Parousia Press.

ELLIOT, John (1994): La investigación-acción en educación. Madrid, Ediciones Morata.

FALS BORDA, Orlando (1991): Action and Knowledge: Breaking the Monopoly. Nueva York, Apex Press.

--- (1979): El Problema de cómo investigar la Práctica y cómo transformarla por la Praxis. Bogotá, Ediciones Tercer Mundo, $2^{\mathrm{a}}$ edición.

GADAMER, H. G. (1984): Verdad y método. Salamanca, Ed. Sígueme. 
Graves, B. (2000): Political Discourse. Theories of Colonialism and Postcolonialism: Deconstruction. Brown University.

HALL, S. (1997): «Forms of Reflective Teaching Practice in Higher Education», en R. Posposisl y L. Wilcoxon: Learning Teaching, pp. 124-131. Murdoch University.

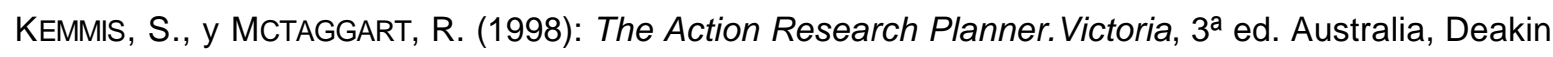
University.

KLAGES, M. (1997): Structure, Sign, and Play in the Discourse of the Human Sciences._Comentarios a J.

Derrida. Boulder, University of Colorado.

MORIN, E. (1999): Los Siete saberes Necesarios para la Educación del Futuro. París, Unesco.

PARRA, C. H. (1995): Dimensión etica de la investigación-acción educativa. Tesis de Doctorado presentada en la Universidad de Navarra. Pamplona.

RESTREPO, Bernardo (2000): «Maestro investigador, Escuela Investigadora e Investigación de Aula», en: Cuadernos Pedagógicos, n.ำ 14. Medellín, Universidad de Antioquia.

SCHON, Donald (1987): «Educating the Reflective Practitioner». Presentation to the 1987 meeting of the American Educational Research Association. Washington, D.C.

--- (1981): Investigación y Desarrollo del Currículo. Madrid, Morata.

STENHOUSE, Lawrence (1993): La investigación como base de la enseñanza. Madrid, Ediciones Morata. WALKER, R. (1971): «The social setting of the classroom: a review of observational studies and research». Tesis no publicada. Universidad de Londres. Chelsea College of science and tecnology.

WITTROCK, M. C. (1989): La Investigación de la Enseñanza. Mexico, Paidós. 


\title{
Contactar
}

Revista lberoamericana de Educación

\author{
Principal OEI
}

